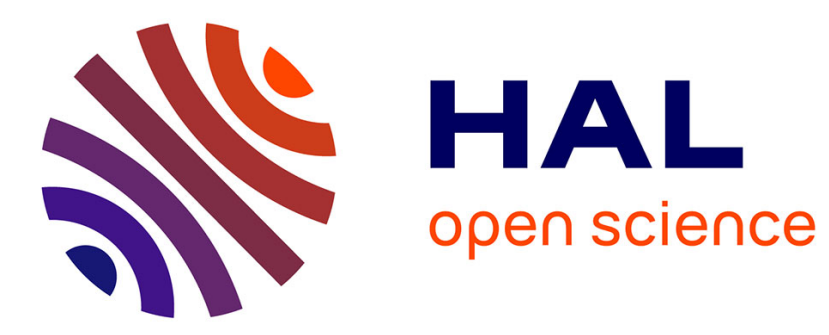

\title{
Classification nominale en ixcatèque
}

Denis Costaouec, Michael W. Swanton

\section{To cite this version:}

Denis Costaouec, Michael W. Swanton. Classification nominale en ixcatèque. La linguistique : revue internationale de linguistique générale, 2015, 51 (2), pp.201-238. halshs-01252034

\section{HAL Id: halshs-01252034 \\ https://shs.hal.science/halshs-01252034}

Submitted on 13 May 2019

HAL is a multi-disciplinary open access archive for the deposit and dissemination of scientific research documents, whether they are published or not. The documents may come from teaching and research institutions in France or abroad, or from public or private research centers.
L'archive ouverte pluridisciplinaire HAL, est destinée au dépôt et à la diffusion de documents scientifiques de niveau recherche, publiés ou non, émanant des établissements d'enseignement et de recherche français ou étrangers, des laboratoires publics ou privés. 


\title{
CLASSIFICATION NOMINALE EN IXCATÈQUE ${ }^{1}$
}

\author{
par Denis CostaOUEC* et Michael SWANTON** \\ *Université Paris Descartes - USPC et Sedyl (CNRS - INALCO - IRD) \\ **Universidad Nacional Autónoma de México - CH \\ denis.costaouec@laposte.net \\ mwswanton@yahoo.com
}

Ixcatec (Popolocan-Otomanguean, Mexico), like other Otomanguean languages of Oaxaca, Mexico, possesses a system of nominal classification for subsets of nouns related to persons, animals, plants, and objects characterized by different formal criteria (length, roundness, etc.), material or function. In this article we seek to establish the functional status of this system. Drawing on Grinewald's typology of nominal classification (1999, 2000), we distinguish class terms, used only in word formation, from noun classifiers, which permit referent tracking in discourse. Our review of the etymological origins of the least transparent classifiers calls into question the assumed time depth of noun classifiers in Popolocan.

\section{INTRODUCTION}

L'ixcatèque (code ISO 639-3 : ixc) appartient à la famille otomangue, groupe popolocan, comme le popoloca, le chocho (ou chocholtèque) et le mazatèque. Il est parlé uniquement dans la commune de Santa María Ixcatlán au nord-est de la région montagneuse appelée Mixteca Alta (État d'Oaxaca, Mexique). Au sein du groupe popolocan, le popoloca et le chocho sont les plus étroitement liés et partagent plusieurs innovations phonologiques alors que le mazatèque est divergeant. On considère que l'ixcatèque occupe une position intermédiaire bien que sa place dans le stemma n'ait pas encore été clairement établie (Hamp, 1958, 1960 ; Veerman-Leichsenring, 2000). C'est une des langues indigènes du Mexique en plus grand risque d'extinction, puisque l'on compte actuellement moins de dix locuteurs, tous du troisième âge ${ }^{2}$. Comme d'autres langues otomangues de l'État d'Oaxaca, l'ixcatèque présente un système de classification nominale, limité à une partie du lexique. Les catégories concernées sont :

- les anthroponymes, les noms de fonctions sociales, les noms des habitants de localités connues (ex. : Ji-tiópá « M. Théophile », ði-ndbà «M. le président», di-f/gè « habitant de Coixtlahuaca », etc. $)^{3}$;

\footnotetext{
${ }^{1}$ L'article est basé sur la communication des auteurs lors du $34^{\mathrm{e}}$ Colloque international de linguistique fonctionnelle, le 30 octobre 2012, Oaxaca, Mexique.

${ }^{2}$ Un programme de documentation de cette langue, dirigé par D. Costaouec et M. S wanton, a été mené entre 2010 et 2013 (MDP 0214 financé par l'Endangered Languages Documentation Programme). Une partie du matériel produit est déposée dans l'Endangered Languages Archive (ELAR), à Londres, et consultable en ligne.

${ }^{3}$ Dans cet article on a retenu une notation phonétique large pour la présentation des exemples. L'ixcatèque possède trois niveaux de tons; les tons haut et bas sont notés par des diacritiques : $\mathrm{H}<$ accent aigu $>, \mathrm{B}<\mathrm{accent}$ grave> ; le ton moyen n'est pas noté. Le complexe constitué d'un marqueur de classification et d'un second élément spécifique forme une seule et même unité lexicale, toutefois dans le présent article on a segmenté le marqueur de classification afin de faciliter la lecture des exemples.
} 


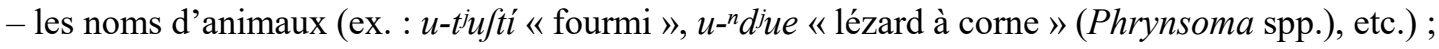

- la majorité des noms de plantes (ex. : tshu-káhà «rose d'Inde»(Tagetes erecta), tshu-ska « millepertuis perforé » (Tagetes lucida), /ka-fhù « fausse ambroisie » (Dysphania ambrosioides), ja${ }^{n}$ ḑatshe « guaje » (Leucanea esculenta), etc.);

On trouve en outre un certain nombre de composés qui se rapprochent des noms classifiés par la régularité et la productivité de leurs procédés de formation :

- des noms de liquides (ex. : ${ }^{n} d a-t h \grave{\imath}$ « sueur », ${ }^{n} d a-t f e$ « mezcal, alcool », etc.) ;

- des noms d'objets caractérisés par un trait formel comme la rotondité (ex. : tiu-t $t^{2} i$ « genou », tiu-hí « grelot», etc.);

- d'autres caractérisés par la matière (ex. : présence de poils, $\int a-f k u$ «cil », $\int a-s k a$ « cheveux », présence de peau ou de cuir, ra-ts?ua «lèvre », ra-tè « sandales », etc.);

- des toponymes caractérisés par une particularité orographique ou hydrographique (ex. : $a i^{n} g i$ « colline » ai $i^{n}$ i-jatfa, «Cerro de Cuatillo », $i^{n} d a ̀$ « eau, rivière » nda-nume «San Pedro Nodón », etc.).

Il n'y a dans cette langue ni « classificateurs génitivaux », ni « classificateurs numéraux » et l'on n'identifie pas non plus de «classificateurs verbaux» ou «classificateurs de prédicat 》 (Allan, 1977). Ce type de classification nominale n'est pas non plus un système de genre ou de classes nominales de type bantou (voir Corbett \& Mtenje, 1987, Creissels, 1999, Rebuschi, 1999, entre autres auteurs) puisque la classification ne concerne qu'une partie seulement des noms et qu'il n'y a pas de répercussions morphologiques sur les unités liées syntaxiquement au nom classifié.

Le présent article tente de délimiter et de caractériser le système de classification nominale qui fonctionne dans la langue ixcatèque. Notre ambition était de porter un regard neuf sur le phénomène en nous appuyant sur les outils de la linguistique générale, en évitant d'isoler la classification nominale des autres caractéristiques importantes de la langue, et notamment de ses procédés généraux de lexicogenèse. L'article passe en revue les différents moyens disponibles pour assurer la classification, en s'appuyant sur les réflexions antérieures sur le phénomène (entre autres Craig, 1986a et b, Grinevald, 1999 et 2000, Aikhenvald, 2000, Senft, 2000, Contini-Morava et Kilarski, 2013) et sur les travaux d'Annette VeermanLeichsenring sur les classificateurs et la coréférence dans les langues popolocanes (Veerman-Leichsenring, 2001, 2004).

Les complexes lexicaux que nous nommons noms classifiés sont constitués au minimum d'un marqueur de classification et d'un second composant spécifique. On s'intéresse essentiellement à leurs procédés de formation, à leurs caractéristiques sémantiques et au phénomène de reprise pronominale que certains d'entre eux autorisent dans l'énoncé. On verra que la classification nominale concerne aussi la morphologie, au sens martinétien du terme, dans la mesure où elle s'impose aux locuteurs pour une partie du lexique. Dans ce qui suit, le terme marqueur de classification, qui reprend la catégorie de classification marker proposée par Contini-Morava \& Kilarski, 2013, désigne tout type d'unités fonctionnant comme premier composant d'un nom classifié et il recouvre donc les termes de classe (c'est-à-dire des noms génériques de la langue employés dans un processus de classification purement lexical) et les classificateurs nominaux (unités spécialisées dont l'emploi a des conséquences au-delà du nom classifié). L'analyse proposée envisage le fonctionnement de la classification nominale dans son ensemble et pas seulement les caractéristiques des «préfixes classificateurs» qui ont surtout retenu l'attention des spécialistes jusqu’à présent (p.ex. Mock, 1977 et Veerman- Leichsenring, 2001).

Nous analysons les faits selon quatre axes :

1) D'un point de vue syntactico-énonciatif, selon que le type de nom classifié autorise ou non sa reprise pronominale dans l'énoncé, ce qui justifie notre distinction entre noms à classificateurs et noms à termes de classe. Une application stricte de ce critère pourrait conduire à limiter le phénomène de classification nominale aux seuls noms qui imposent la reprise pronominale (désignation des humains et des animaux), mais ce choix laisserait de côté de nombreux problèmes, touchant notamment à la création lexicale, qui doivent être pris en compte.

2) En distinguant les noms classifiés construits en discours (essentiellement ceux qui servent à désigner les humains) de ceux qui sont fortement lexicalisés. 
3) En caractérisant les différents procédés de formation des noms classifiés : d'une part en faisant la différence entre le procédé spécifique de création des noms classifiés (marqueur de classification + lexème toujours conjoint) et les autres procédés synthématiques qui peuvent également être sollicités et, d'autre part, en repérant les possibilités de commutation, concaténation et juxtaposition des marqueurs de classification.

4) Enfin, d'un point de vue sémantique, en montrant que différents types de rapports peuvent s'établir entre le marqueur de classification et le second composant.

Le corpus étudié est fourni par les dictionnaires établis par les auteurs à partir des travaux de María Teresa Fernández de Miranda (1961) et de leurs propres recherches, notamment l'analyse de textes des années 1950 publiés dans Miranda (1961) et de textes recueillis récemment dans le cadre du projet de documentation de la langue. On a aussi puisé dans le matériel des enquêtes ethnobotaniques menées depuis plusieurs années par Selene Rangel Landa et ses collègues du Centro de Investigaciones en Ecosistemas (CIEco UNAM) sur le territoire de Santa María Ixcatlán, en particulier pour la dénomination scientifique des espèces végétales.

Il faut signaler qu'à la différence d'autres langues de la région, y compris le chocho et le popoloca, il n'existe pas pour l'ixcatèque de corpus de textes notés en caractères latins par des religieux catholiques ou des indigènes éduqués durant la période coloniale. Les documents les plus anciens connus à ce jour datent du XIX ${ }^{\mathrm{e}}$ siècle : deux traductions du Notre Père de 1854 et de 1888 et des vocabulaires de 1887 et 1893 (voir Swanton, 2008, pour une description des premiers écrits ixcatèques). Roberto Weitlaner a noté trois récits en 1939 avec les locuteurs Agapito Jiménez, Doroteo Jiménez et Amado Castro, et un autre en 1948 en collaboration avec Casiano Jiménez et Tomás Martel. En 1946, Helen Black a recueilli un texte auprès de Francisca Guzmán. María Teresa Fernández de Miranda a publié sept textes, tous produits par le même locuteur, Doroteo Jiménez, qui s'était rendu à Mexico durant trois mois pour travailler avec la linguiste. En 1950, le même Doroteo Jiménez en collaboration avec des linguistes-missionnaires de l'Instituto Lingüistico de Verano a rédigé une brochure élémentaire sur le vocabulaire ixcatèque et donné une traduction de certains passages de la Bible. Annette Veerman-Leichsenring a consacré quelques articles à la langue et Jojan van Zantwijk, une de ses étudiantes, a enquêté à Santa Maria Ixcatlán pendant quelques mois fin 1996 - début 1997, mais ces travaux n'ont pas produit de textes en ixcatèque. Ainsi, malgré le nombre dramatiquement réduit de locuteurs avec lesquels il est possible de travailler aujourd'hui, nous disposons grâce au programme de documentation de la langue, qui a produit près de 60 heures d'enregistrement en partie analysées, d'une quantité de matériel sans commune mesure avec ce qui existait auparavant.

L'article est organisé comme suit: la première partie présente les caractéristiques de la classification nominale dans les langues popolocanes dans les travaux de nos devanciers et relève des problèmes non résolus ; la deuxième caractérise les termes de classe ; la troisième s'intéresse au cas particulier des toponymes ; la quatrième détaille le système de classificateurs nominaux ; la cinquième établit la distinction nécessaire entre noms classifiés intégrés au lexique et noms classifiés formés en discours ; la sixième s'intéresse à l'origine des classificateurs nominaux humains; la conclusion résume les résultats de l'analyse et suggère que l'approche adoptée peut amener à de nouvelles pistes de recherche quant à l'organisation et à l'ancienneté de la classification nominale dans les langues popolocanes.

\section{QUELQUES CARACTÉRISTIQUES DE LA CLASSIFICATION NOMINALE DANS LES LANGUES POPOLOCANES}

Rappelons en premier lieu quelques propriétés générales de ce que l'on nomme classification nominale. Comme le signale Leonard Talmy (1992:131), les langues « subcatégorisent grammaticalement les noms selon certains paramètres sémantiques, principalement propre/commun, comptable/massif, unitaire/collectif, relatif/absolu... » ${ }^{4}$ et Joseph Greenberg explique cette propriété par le fait que «...nouns are continuing discourse subjects and are therefore in constant need of referential devices of identification » (Greenberg 1978: 78). La recherche d'une meilleure adéquation de la désignation aux propriétés des référents, telles qu'elles sont perçues, ce que Greville Corbett (1991: 322) nommait reference tracking,

\footnotetext{
${ }^{4}$ Notre traduction.
} 
serait donc au principe de la tendance à catégoriser. On peut ajouter de nombreux autres paramètres à la liste de Talmy : possession aliénable/ inaliénable, humain/non humain, tout/partie du tout, sexe, propriétés formelles, aspects fonctionnels, statut social des humains, etc. Par ailleurs, la classification nominale peut concerner tous les noms d'une langue (les langues à genres, les langues à classes nominales de type bantou) ou être limitée à une partie d'entre eux, ce qui concerne précisément l'ixcatèque. Elle peut être d'emploi général ou circonscrite au contraire à certains contextes, par exemple à la numération (classification numérale, dans des langues comme le chinois ou le thaï, par exemple) ou encore à l'expression de la possession (langues uto-aztèques, entre autres). Comme l'ont montré de nombreuses études, différents systèmes de classification peuvent coexister au sein d'une même langue (pour les langues amérindiennes, voir Craig, 1986a, De León, 1987, Zavala, 2000). Enfin, la classification nominale peut être liée à divers phénomènes «d'accord», de coréférence ou de reprise pronominale dans l'énoncé. Les situations observées dans les langues sont variées et la classification nominale met souvent en œuvre diverses ressources linguistiques, réunies en un système assez hétéroclite, ce qui témoigne de sa construction progressive à partir du matériel disponible dans la langue. On verra que c'est le cas pour l'ixcatèque.

\section{1 Études antérieures dans le domaine popolocan}

L'existence de systèmes de classification nominale a été signalée pour les branches de langues mixtèques, popolocanes, zapotèques, amuzgo et chinantèques de la famille otomangue ainsi que les langues mayas du groupe q'anjob'alan. Dans le domaine popolocan, le phénomène a d'abord été identifié en chocho et en popoloca : Francisco Belmar $(1899: 16 ; 1921$ : 204) signale les prefijos «préfixes » employés pour les noms d'animaux (cho. $u$-, pop. $k u$-) et c'est à Pablo González Casanova (1925: 516) que l'on doit les premières observations sur l'ixcatèque lorsqu'il identifie le préfixe $u$ - employé pour «clasificar los nombres de animales ». En mazatèque le phénomène est moins marqué et la langue reste un peu en marge de ce type d'études. Au milieu du $\mathrm{XX}^{\mathrm{e}}$ siècle ont été menées les premières reconstructions systématiques de la famille otomangue et de ses branches. Sarah Gudschinsky (1956) et Paul Kirk (1966) ont reconstruit le proto-mazatèque et Gudschinsky (1959), en s'appuyant sur les travaux de Fernández de Miranda (1951), a reconstruit le proto-popolocan. Ces études diachroniques ont identifié et reconstruit plusieurs classifier morphemes. Ainsi, Gudschinsky (1959: n. 66) propose-t-elle les protoformes *ku et *ču, qu'elle glose comme « animal », en se basant sur les marqueurs de classification de l'ixcatèque, du popoloca, du chocho et du proto-mazatèque. Toutefois, ces travaux de reconstruction s'intéressaient principalement à établir la filiation et la relation entre les formes, tandis que le fonctionnement des classificateurs restait en grande partie inexploré. À partir des années 1970, la particularité des systèmes de classification des langues de Mésoamérique est régulièrement signalée (Kilarski, 2013) et, dès lors, les travaux sur les langues popolocanes fournissent des listes structurées de «classificateurs» (généralement considérées comme fermées) ${ }^{5}$. Ces études ont établi l'habitude de considérer le terme de classification comme un préfixe qui indique la catégorie sémantique à laquelle appartient le nom classifié ${ }^{6}$.

Annette Veerman-Leichsenring a mené une étude poussée de la classification nominale popolocane avec trois objectifs principaux :

a) Donner des inventaires structurés et exemplifiés des classificateurs popolocans organisés selon un critère sémantique, en opposant les traits « humain » (modulé selon les variantes socioculturelles) et «non-humain ». Elle distingue également un ensemble de noms qu'elle appelle "spécifiques » car ils ne sont pas classifiés (le terme specific class a été proposé par Craig, 1986a, pour les désigner);

b) Décrire le fonctionnement de ce qu'elle appelle «coréférence » liée à certains noms classifiés. Elle décrit ce système essentiellement à partir d'exemples du popoloca de Metzontla et du chocho

\footnotetext{
${ }^{5}$ Mock (1977 : 39), Stark (1978 : 21-22), Veerman-Leichsenring (1984:89-91, $1991: 82,1992,2000: 22-24$, 2001c : 330-332), Austin et al. (1995: 305-307).

${ }^{6}$ Belmar (1921 : 204), González Casanova (1925 : 516), Swadesh (1960 : 89-90), Veerman-Leichsenring (1992 ; 2001b : 337; 2004 : 416).
} 
d'Ocotlán, mais elle observe qu'un tel phénomène, quoique moins productif, existe aussi en ixcatèque et en mazatèque ${ }^{7}$;

c) Proposer quelques explications historiques à l'apparition des différents classificateurs et marques de coréférence dans les langues popolocanes. Elle reconnait que les classificateurs trouvent leurs origines dans des noms génériques et fait l'hypothèse qu'il s'agit d'un système ancien dans les langues popolocanes qui a perdu par la suite de sa productivité dans certaines langues filles ${ }^{8}$.

On reproduit ci-dessous l'inventaire des «classifiers » établi par Veerman-Leichsenring (2004: 428) pour l'ixcatèque :

Tableau 1: Classificateurs de l'ixcatèque selon A. Veerman-Leichsenring (2004: 428)

\begin{tabular}{|c|c|}
\hline $\begin{array}{l}\text { Human : } \\
m i^{2} \text {-'person, people' } \\
d i^{2} \text {-'familiar male' } \\
k u a^{2} \text {-'familiar female' } \\
n a^{2} \text {-'reverential female' } \\
n a^{2} ? m i^{2}-\text { 'reverential male' } \\
2 n n_{3} e^{I} \text {-'child' }\end{array}$ & $\begin{array}{l}\text { Specific classifiers : } \\
\tilde{n} u^{2} \text {-'corn product' } \\
h m a^{2} \text {-'bean' } \\
h \tilde{n} a^{2} \text {-'chili pepper' } \\
\tilde{c} u \text {-'squash' } \\
\tilde{s} \tilde{a}^{2} \text {-'pulque' } \\
\tilde{c} \tilde{i}^{2} \text {-'zapote' }\end{array}$ \\
\hline 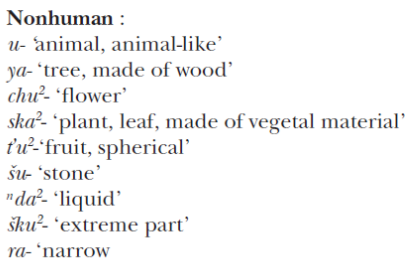 & \\
\hline
\end{tabular}

\subsection{Quelques problèmes non résolus}

Malgré les éclairages importants apportés par nos devanciers, des questions non résolues demeurent. Quels sont, par exemple, les critères qui permettent d'identifier un système de classification nominale ? Annette Veerman-Leichsenring propose une liste dont on verra qu'elle mêle des véritables noms classifiés à classificateurs nominaux, des noms classifiés à termes de classe et des composés «ordinaires ». Cela s'explique par le fait qu'elle n'utilise qu'un seul critère, la présence d'un des classifiers qu'elle recense, qui sont en fait des unités à statut très variable. En effet, sur quelle base considérer qu'un monème donné est un classificateur? Annette Veerman-Leichsenring écrit à ce propos :

« The different morphemic values of the classifying elements display a development from free nouns to classificatory prefixes and finally to fully fossilized elements that can no longer be isolated as free morphemes. Different phases of this development are present in the modern Popolocan languages, which sometimes makes it difficult to define the morphological status of the classifier » (VeermanLeischenring, 2004 : 416).

Elle signale trois caractéristiques de la classification nominale qui sont a) l'attrition phonétique du marqueur de classification, b) le figement du nom classifié où il n'est pas possible d'insérer un déterminant et c) la possibilité de changements de sens quand le classificateur est omis'. Ces critères doivent être pris en considération, mais ils ne permettent pas de faire le départ entre les classificateurs véritables et d'autres types d'unités ayant la même fonction dans les noms classifiés, et partant, entre procédé spécifique de classification et composition ordinaire.

\footnotetext{
${ }^{7}$ Veerman-Leichsenring (2004: 419, cf. $1984: 125-126 ; 1991: 80-92 ; 1992 ; 2000: 74-76 ; 2001 \mathrm{~b} ; 2001$ changes). Austin et al. (1995: 308) mentionnent aussi ce type de coréférence pour le popoloca d'Atzingo et parlent de «fonction pronominale des noms génériques ».

${ }^{8}$ Veerman-Leichsenring, 2004, cf. $1992: 9$. Cette interprétation est dans la lignée de travaux contemporains sur d'autres langues mésoaméricaines, comme par exemple le mixtèque (León Pasquel, 1987, 1988, Macaulay, 1996, voire aussi Veerman- Leichsenring, $1994: 214$ ).

${ }^{9}$ Veerman-Leichsenring, $2004: 417-18$. Austin et al. (1995: 305-307) considèrent ces classificateurs en popoloca d'Atzingo comme des noms génériques entrant dans la composition.
} 
Il faut donc s'interroger sur l'extension réelle du système de classification et savoir si l'inventaire de Veerman est justifié.

Un autre problème est de savoir si la classification nominale ixcatèque est active en synchronie ou si nous n'avons affaire qu'à un « vestige de classificateurs » (Veerman-Leichsenring, $2004: 448$ ). Enfin, se pose la question de l'ancienneté du système de classification nominale dans les langues popolocanes. Les propositions de reconstruction de Veerman-Leichsenring, limitées à quelques unités, s'appuient en partie sur des jeux de correspondance qui ne sont pas attestés dans les travaux antérieurs sur cette famille. On peut s'interroger sur la possibilité de reconstruire les classificateurs humains au-delà du chocho-popoloca. Nous tenterons de proposer quelques pistes de réflexion sur ces questions.

\section{TERMES DE CLASSE IXCATÈQUES}

L'ixcatèque possède une série de noms génériques, utilisables en tant que tels en énoncé, qui participent systématiquement à la lexicogenèse dans certains domaines sémantiques mais n'ont pas alors de rôle syntaxique ou pragmatique. Selon la typologie des classificateurs de Grinevald, nous appelons ceux-ci termes de classes (1999: 106-108; 2000 : 59-61).

Il s'agit des noms qui restent actifs dans la langue, généralement d'unités monosyllabiques qui sont employées sans modification en tant que termes de classe. L'exception apparente du changement de ton et de la quantité vocalique, comme pour le nom jà [jà:] « arbre » dont le ton bas devient moyen, résulte d'une contrainte phonologique bien identifiée dans la langue (Miranda, 1959, DiCanio, article soumis) qui interdit le ton bas à l'initiale des mots : les voyelles à ton bas des unités monosyllabiques sont plus longues que les voyelles à ton moyen ou haut, mais un tel allongement ne se produit jamais en position pénultième dans les polysyllabiques ; par conséquent, la quantité vocalique est réduite en position non finale (DiCanio, soumis). Deux noms bisyllabiques fikà «herbe, feuille ", indà « eau » et ruwa "peau » subissent une réduction phonétique, principalement par chute d'une voyelle interne ou initiale. Le phénomène ton bas $>$ ton moyen se produit également dans $i^{n} d \grave{a}$ 《eau » $>{ }^{n} d a$ - «liquide ». Le nom ${ }^{n} d \xi i j a ~ « q u e l i t e »$ (désigne les plantes herbacées comestibles du genre Anoda cristata, Justicia spicigera, etc. ${ }^{10}$, qui entre dans un nombre réduit de complexes, semble se maintenir sous sa forme bi-syllabique, mais en tempo rapide des variantes monosyllabiques sont enregistrées, et dans le cas du "papaloquelite» (Porophyllum punctatum et Porophyllum ruderale) c'est cette variante qui s'est lexicalisée: ${ }^{n} d \zeta e-h n i ́\left(<{ }^{n} d \zeta i j a-h n i ́\right.$, «quelite savoureux »).

Les noms classifiés avec des termes de classes sont toujours inanimés. Ils se présentent dans trois champs sémantiques principaux -flore, anatomie et matériel/forme- mais on note quelques chevauchements en raison de l'extension sémantique du terme de classe. Par exemple le terme désignant un « arbre » est aussi utilisé pour désigner des objets en bois (en général des artefacts) et le terme de classe pour « fruit » peut être utilisé en référence aux choses sphériques, incluant les parties du corps. Sur la base de la productivité et de l'hétérogénéité, nous adoptons la distinction que fait Craig (1986a : 277) entre termes « généraux » et " spécifiques ». Alors que certains termes de classe (p.ex. ja, tiu, $f k a$ ) sont communs et apparaissent dans des douzaines (voire des centaines) de noms, d'autres termes (p.ex. t ${ }^{\prime} u,{ }^{n} d \xi i j a, t^{j} a h u$ ) ont un référent plus spécifique et n'apparaissent que dans quelques noms seulement.

Tableau $2:$ Les termes de classe ixcatèques principaux par champ sémantique

\begin{tabular}{|c|c|}
\hline 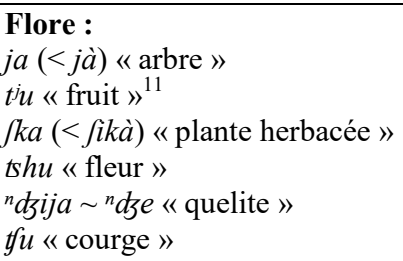 & 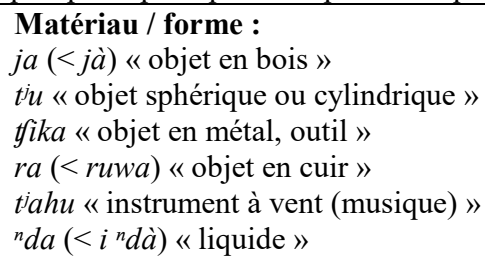 \\
\hline $\begin{array}{l}\text { Anatomie : } \\
d^{j} a\left(<i^{n} d^{j} \grave{a}\right) \ll \text { os, jointure » }\end{array}$ & \\
\hline
\end{tabular}

\footnotetext{
${ }^{10}$ Sur les « quelites » dans la classification de vie mesoaméricaine, voir De Avila, 2010.

${ }^{11}$ Comme unité syntaxique autonome $t^{i} u$ fait référence à « oeuf » et timí fait référence à « fruit ».
} 
ra $(<$ ruwa $) \ll$ peau »

$t^{i} u$ « partie corporelle sphérique »

Ce type de classification nominale est productif comme on peut le constater dans la domestication des mots empruntés à l'espagnol :

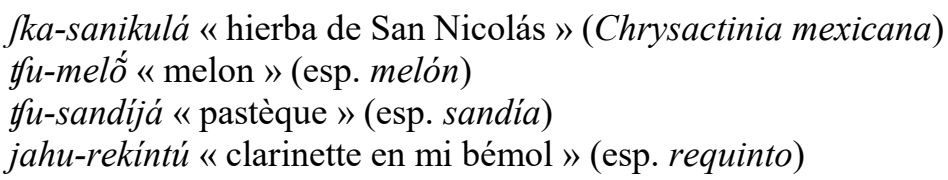

La possibilité existe de recourir à n'importe quel nom générique comme terme de classe quand cela est nécessaire :

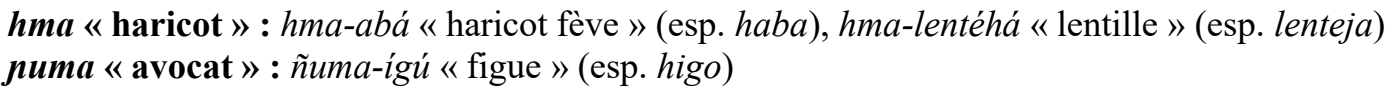

Les termes de classe ne sont donc pas une sous-classe fermée de noms, mais plutôt ce que Rosemary Beam (en préparation), étudiant un phénomène semblable en zapotèque, appelle une sous-classe « semi-fermée ». La liste des termes de classe ixcatèques doit donc être comprise comme un épiphénomène d'un processus productif de création lexicale plutôt que comme un système fermé.

La grande majorité des noms des champs sémantiques concernés par la classification portent systématiquement un terme de classe. Il y a cependant d'importantes exceptions. Dans le champ de la flore, il faut noter que les plantes de grande importance économique et culturelle sont désignées par des noms simples. On peut signaler les noms suivants : nahme «maïs », hma " haricot», hna «piment», tsu « maguey » (agave), thẽ « palmier » (Brahea spp.), $n u^{n} d a$ « nopal » (Opuntia spp.); s’y ajoute une série de noms de végétaux courants : t??ẽ « filles de l'air» (Tillandsia spp.), tsuku « lechuguilla » (Hectia spp.), jutu « biznaga » (Ferocactus spp.), "djihi " herbe », etc. Parmi les termes anatomiques, beaucoup de ceux qui désignent ce que l'on pourrait appeler les parties du corps basiques n'ont pas de terme de classe, p. ex. tsha « veine/tendon », ska « tête », titithu « nez », ţ?ua «bouche », tsathẽ «front », nihna « langue », na?nu « dent», tfa? ã «bras/épaule », síhi «pied», rha «main» et thêe «sang»" ${ }^{12}$. Néanmoins la majorité des expressions désignant les parties du corps imposent l'emploi d'un terme de classe.

\subsection{Procédés de création des noms classifiés à terme de classe}

Un grand nombre de noms classifiés se présentent sous la forme d'un complexe associant un marqueur de classification et un second composant qui n'apparaît que dans ce type de construction et n'appartient à aucune classe syntaxique de la langue. Par exemple le monème ftuhù fait référence à une arme d'épaule, généralement un fusil de chasse. Il n'apparaît jamais indépendamment mais toujours avec un terme de classe. Ainsi avec le terme de classe $j a$ « objet en bois » est formé ja-ftuhù « arme d'épaule », alors qu'avec le terme t'u "objet sphérique », on obtient $t^{i} u-f t u h \grave{u}$ qui signifie «balle». Ce second composant pourrait être rapproché de ce que l'on appelle la « racine » du nom classifié dans les langues bantoues par exemple, (voir Corbett \& Mtenje, 1987, Creissels, 1999, Rebuschi, 1999, entre autres auteurs), c'est-à-dire un segment porteur de sens mais qui ne peut apparaître en discours qu'accompagné d'un marqueur de classe sémantique. On propose d'appeler lexèmes conjoints (en d'autres termes «toujours conjoints ») ces unités de la langue qui ne fonctionnent qu'en tant que composants de noms classifiés et sont proches de ce que Martinet appelait confixes (Martinet, 1979 et 1985 notamment) ${ }^{13}$. Le reste des noms classifiés se partagent

\footnotetext{
${ }^{12}$ En ixcatèque, comme dans d'autres langues mésoaméricaines, le sang n'est pas ouvertement classifié comme un liquide.

${ }^{13}$ Le terme « lexème conjoint » a été préféré à celui de « confixe », plus spécifique, à la suite de discussions avec des collègues fonctionnalistes : merci à C. Clairis et F. Guérin notamment. Voir aussi les « cranberry morphs », cf. Aronoff, 1976.
} 
entre composés «ordinaires » dont le deuxième composant est une unité syntaxique du type Nom ou Adjectif. Le second composant peut être un emprunt à l'espagnol. Exemples :

\section{Type Terme de classe + Lexème conjoint}

- ja : ja-thẽ « genévrier» (Juniperus flaccida), $j a{ }^{n}{ }^{n} g e$ « encino» (principalement Quercus spp.), jaftuhì « arme, fusil », ja-tihu « escalier », etc.

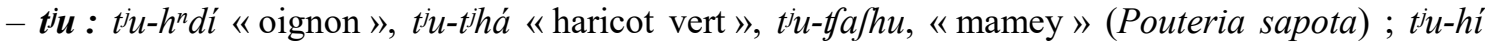
« grelot », tiu-kubi (+poss) «(son) nombril », tiu-sĩ « nuque, cerveau », tiu-ftuhì « balle », etc.

- Jka : Jka-nuftù «menthe»(Mentha × piperita), $\int k a-\int h \grave{u}$ «fausse ambroisie, thé du Mexique» (Dysphania ambrosioides), Jka-fé?è « malamujer» (Cnidosculus tehuacanensis), etc.

- tshu : tshu-káhà « rose d'Inde » (Tagetes erecta), tshu-tyà « orchidées » (Laelia sp.), etc.

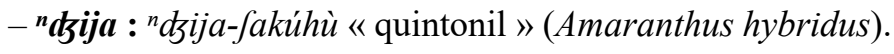

- tfu : tfu-?mi « chayote » (Sechium edule), tfu-kihi « banane ».

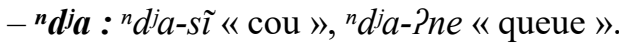

- ra : ra-tshũ « oreille», ra-kutu « vagin».

\section{Type Terme de classe + Nom ixcatèque}

Il s'agit de complexes formés d'un nom employé comme terme de classe et d'un nom ixcatèque pouvant apparaitre seul dans d'autres contextes. En voici quelques exemples :

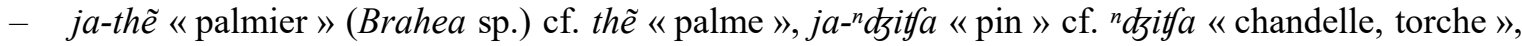

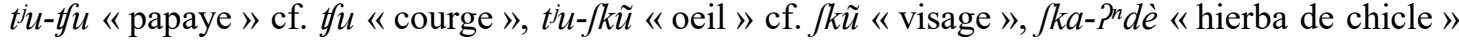
(Euphorbia macropus) cf. Pndè "gomme», Jka-si ndiù « hierba de los ángeles » (Ageratina mairetiana) cf. tsi " diù « vent », tshu-tsikù « senna » (Senna holwayana) cf. tsikù «monnaie », tshu-

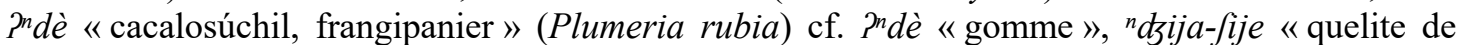
manteca » cf. Sije « graisse », tfu-hná « courge de Siam» (Cucurbita ficifolia) cf. hná « montagne,

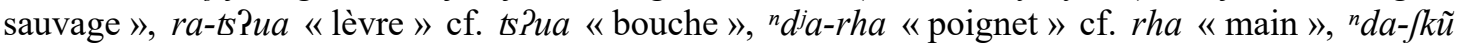
« larme » cf. $\int k \tilde{u}$ « visage ».

\section{Type Terme de classe + Adjectif}

- On y trouve notamment: ja-sé «tringle» cf. sé «mince», tiu-sí « agrumes, prototypiquement

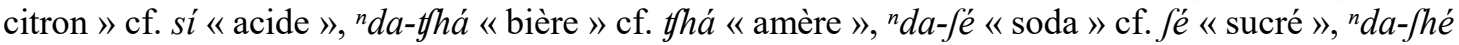
« rivière en crue » cf. Jhé «gros », tika-sé «fil » cf. sé « mince», tiahu-tíjé « clarinette» cf. tíjé «noir ».

\section{Type Terme de classe + Nom espagnol}

- Les noms concernés apparaissent dans les classes $j a, t^{i} u, \int k a,{ }^{n} d z i j a, t f u$ et ${ }^{n} d^{j} a$. On y trouve par exemple : ja-filá « chaise » cf. esp. silla, ja-mulátú «arbre mulâtre» cf. esp. mulato, túráfnú « pêche » cf. esp. durazno, t’u-sarampiố « rougeole » cf. esp. sarampión, Jka-tfapúli « cachapule, diente de león, ixpule» (genre de pissenlit), ndzija tintonil, «tintonil» (Husticia spicigera), tfu-

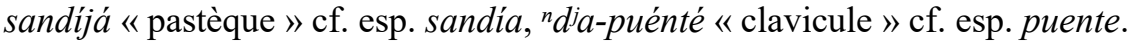

\section{Type Terme de classe + Adjectif espagnol}

- Procédé peu productif: un nom de plante, Jka-higánté «plante géante (Micotania glauca)»cf. esp. gigante.

Le corpus présente un certain nombre de complexes à trois composants ou plus intégrant un nom classifié, ce qui permet d'introduire des distinctions entre référents apparentés. Ces composés sont une manière commode de distinguer des taxa spécifiques face à un taxon générique parmi les formes de vie ${ }^{14}$. Par exemple, ja-thhẽ fait référence au " genévrier » (Juniperus flaccida); cependant, ja-thẽ - ${ }^{n} d a ̀$, littéralement " genévrier d'eau », fait référence au Taxodium huegelii (en espagnol, sabino), tous les deux faisant partie de la famille des Cupressaceae. Le mot $j a{ }^{n}$ ge est un terme générique pour un certain ensemble d'arbres,

\footnotetext{
${ }^{14}$ Sur la distinction entre les taxa génériques et spécifiques, voir Berlin, 1992 et ses références.
} 
principalement ceux du genre Quercus, mais ja- ${ }^{n} g e-t \hat{a}$, " chêne compact, dur ", désigne spécifiquement l'encino amarillo (Quercus liebmannii, Quercus lacta).

Un autre procédé de construction de ces complexes est la concaténation de plusieurs termes de classe dans une séquence linéaire. Souvent la concaténation des termes de classe implique une relation méronymique dans laquelle le terme de classe préposé spécifie une partie de l'objet nommé. Exemples : ra-tiu-fkũ « paupière », thu-jkũ « oeil » Jka-ja-tsi « feuilles du Quercus acutifolia ou Quercus conspersa » (esp. encino colorado), ja-tsi « Quercus castanea », ja-t'u-ská « arbre fruitier chupandía » (Cyrtocarpa procera), t'u-ská « fruit chupandía », Jka-tiu-fti « plant de tomate », tiu-fti « tomate ».

C'est un processus récursif, car un troisième terme de classe préposé peut être introduit si une plus grande spécification méronymique est nécessaire. Ainsi, pour désigner les feuilles du chupandía (Cyrtocarpa procera), il est nécessaire de spécifier que ce sont les feuilles de l'arbre du chupandía (/ka-ja-tiu-ská), et pour désigner la fleur de tomate, il est nécessaire d'indiquer qu'il s'agit de la fleur du plant de tomate (tshu$\left.\int k a-t^{i} u-f t i\right)$. On observe des contraintes de constructions, puisque tshu-fka-tiu-fti « fleur de plant de tomate » est possible, mais pas $*_{t s h u-t j u-f t i *}^{*}$ «fleur de tomate ». Dans quelques cas, la concaténation des termes de classe est complètement lexicalisée : fka-tiu-fije «plante de ricin » (Ricinus communis), de fije «gras », fka$t^{t} u-n u^{n} d u$ « mauve» (Malva parvifolia), de $n u^{n} d u$ « visqueux», tshu-tiu- ${ }^{n}$ dika «lantanier» (Lantana camara), de ${ }^{n}$ dika « maïs grillé », ja-tiu-fté « fromager, kapokier » (Ceiba aesculifolia subsp. parvifolia) (en

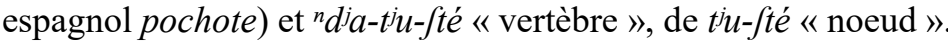

Cette facilité à créer de nouveaux complexes est une particularité de la composition en général mais le procédé a des limites et il faut pouvoir distinguer entre les constructions qui relèvent encore de la synthématique et celles qui sont déjà des syntagmes. Le critère retenu en première analyse est celui du degré de figement du complexe et la possibilité ou non d'y insérer un élément, par exemple un possessif. On constate que les noms classifiés se comportent de ce point de vue comme les noms simples et que le possessif est suffixé au complexe, sans possibilité d'insertion :

- Nom simple + POSS1SG : síhi-nánà « mon pied », tuthì-nánà « ma fourche », etc.

- Complexes à plusieurs composants + POSS1SG : tika-sé-nánà «mon fil de fer », tiu-meló-nánà " mon melon », hma-lentehá-nanà « ma lentille », Jka-tiu-fijé-nánà « mon plant de ricin », etc.

Toutefois certains cas interdisent la postposition du possessif. Les causes de cette contrainte ne sont pas encore bien cernées. On pourrait y voir une forme de classification génitivale, mais le caractère exceptionnel de ces structures, limitées à quelques noms classifiés, interdit de postuler un tel système en ixcatèque. Exemples: hmá-nánà hma ménò «mon haricot de milpa» (champ où est cultivé le maïs conjointement à certaines variétés d'haricots), litt. «mon haricot, haricot de milpa » hmá-nánà hma uhè « mon haricot noir», litt. « mon haricot, haricot noir» dsijá-nánà ndbija fije « quelite de manteca» litt. « ma quelite, quelite de manteca » Le même principe s'observe dans certaines constructions très complexes du type $\int k a-n u f t u ́-n a ́ n a ̀ ~ J k a-n u / t u$-é u-kútfi « mon chepito » (Clinopodium mexicanum), litt. « ma menthe, sa menthe du cochon (c-à-d. chepito) ».

\subsection{Noms classifiés et syntagmes possessifs}

Il faut signaler ici le jeu intéressant entre les complexes lexicaux que nous étudions et des structures possessives qui contribuent elles aussi à la lexicogenèse. C'est le cas par exemple des oppositions possibles entre un composé comme $\int k u ́$-jà « sommet d'un arbre » (litt. « pointe arbre ») et un syntagme possessif figé comme $\int k u$-é jà « écharde » (litt. « sa pointe de l'arbre »), ou encore entre un nom classifié du type $/ k a-j a-$ tsi « feuille de chêne rouge » et une structure possessive du type $\int k$-é jà « feuille d'arbre (en général) » (litt. « sa feuille de l'arbre »). En revanche, d'autres exemples attestent d'un signifié similaire pour le figement avec possessif et le composé : cf. ru-é jà " écorce » (litt. "sa peau de l'arbre »), face à ra-jà : "écorce » (litt. « peau arbre »), construit sur le même modèle que $r{ }^{n}{ }^{n} d_{\zeta i s} \tilde{e}$ « courroie, fronde » (litt. " peau feuille de maguey »).

\subsection{Noms classifiés à termes de classe et composition}

Les complexes du type Terme de classe + Nom ou Terme de classe + Adjectif (unités appartenant à des classes syntaxiques bien identifiées) sont donc des composés « classiques », résultant d'un procédé courant dans la langue. Le problème théorique posé est celui des critères qui permettent de distinguer les noms 
classifiés à terme de classe d'autres types de noms complexes construits par composition. La question qui se pose aussi bien pour l'ixcatèque que pour d'autres langues otomangues (voir par exemple Antonio Ramos, 2013 pour le zapotèque de San Pedro Mixtepec). Selon nous, il y a classification nominale en ixcatèque quand un procédé de création lexicale devient systématique (création de paradigmes à partir d'une série de composants initiaux : ce que Dixon $(1965,1982,1986)$ et Allan (1977) nommaient une overt indication des classes), qu'il s'impose à toute création lexicale dans un champ sémantique donné, qu'il s'étend à des complexes à lexèmes conjoints et qu'il est majoritairement du type hyperonyme + hyponyme. Du point de vue diachronique, la généralisation sémantique (ce qui correspond à une généralisation des contextes d'usage) que certains des termes de classe ont subi renforce cette interprétation. Par exemple, alors que le nom jà fait référence prototypiquement à «arbre », son utilisation dans la classification étend ses référents à n'importe quel objet fait principalement de bois.

\section{LE CAS DES TOPONYMES}

L'enquête sur les toponymes ixcatèques est encore embryonnaire mais certaines régularités sont observables, qui poussent à rapprocher leur structure de celle des noms classifiés. En effet, à côté de noms simples ou inanalysables en synchronie, minoritaires dans le corpus, on rencontre beaucoup de noms complexes qui peuvent être des noms classifiés, parfois de longs figements ou encore des combinaisons de termes de classe et de lexèmes conjoints.

Exemples de toponymes simples ou inanalysables :

- thui «la ville d'Oaxaca », nunírẽ «Quiotepec », tuth uั̃ «Tamazulapan », faruwà « la ville de México », etc.

Il s'agit toujours de toponymes majeurs (Lázaro Carreter, 1968 [1953] : 395), désignant des villes importantes de la région ou la capitale fédérale. Les toponymes mineurs et la microtoponymie sont souvent des complexes à terme de classe.

Exemples de toponymes complexes à terme de classe :

- hnà « montagne » : hna-jè "Cerro Culebrón », hna-katúse «Cerro Catuse », hna-tikate «Cerro Iscate », etc.

- ${ }^{n} \boldsymbol{d a}$ « eau, rivière » ${ }^{n} d a-{ }^{n}$ giwà « Río Camino de Coixtlahuaca », ${ }^{n} d a-n u m e$ « San Pedro Nodón », ${ }^{n} d a-t i j e ́$ « Puebla », ${ }^{n} d a-{ }^{n}$ gia?jà « Río Seco », ${ }^{n} d a-t s i^{n} g a f e ~ «$ Río Frío », ${ }^{n} d a-n u f u ́ u$ « Agua del Perro », ${ }^{n} d a-{ }^{n}$ gita « Río Vela », etc.

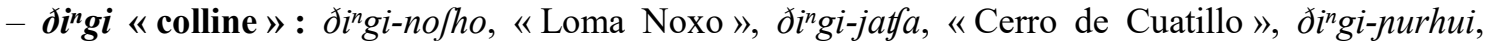
« Gandudito », ðingi-frenià « Malpaso » (litt. « colline d'Eufrenia »), etc.

- tsaka « ravin » : tsaka-katúse, « Barranca Catuse », tsaka-ndaðétfi, «Barranca Ndadechi », etc.

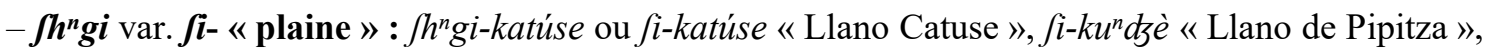
$\int h^{n}$ gi-tikate « Llano Iscate », etc.

L'analyse détaillée de ces complexes reste à faire, notamment pour comprendre le détail des procédés de création à l'œuvre, en relation avec les réalités topographiques, les emprunts aux langues voisines ou à l'espagnol.

\section{CLASSIFICATEURS NOMINAUX IXCATÈQUES}

Une deuxième série de marqueurs de classification ixcatèques participe à la lexicogenèse et exprime des catégories sémantiques générales. Ces marqueurs diffèrent des termes de classe de plusieurs façons. Tout d'abord, ils forment une classe fermée de marqueurs de classification qui indiquent des catégories animées, alors que les termes de classe sont une classe semi-fermée et renvoient à des catégories inanimées. Ensuite, contrairement aux termes de classe qui permettent la concaténation avec d'autres termes de classe, l'ensemble des marqueurs de classification animés n'autorise pas ce procédé de création lexicale. Enfin, les termes principaux de cette classe fermée impliquent une reprise pronominale à la troisième personne, ce qui 
permet la continuité référentielle dans le discours. En suivant la typologie de Grinevald (1999, 2000), on nommera classificateurs nominaux ces unités dont l'emploi à des répercussions dans la phrase, au-delà du nom lui-même.

Il y a trois principaux classificateurs nominaux en ixcatèque : $\delta i-$ "personne de sexe masculin », $k^{w} a-$ «personne de sexe féminin» et $u$ - «animal». Ils sont productifs et sont fréquemment utilisés avec des emprunts à l'espagnol. Exemples : đi-fiská «intendant de l'église» (esp. fiscal), ði-erérú «forgeron»

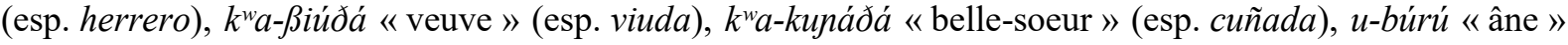
(esp. burro), u-pátú « canard» (esp. pato).

Les classificateurs nominaux humains marquent :

- la classe d'âge pour les jeunes (ex. : đi-ítì «garçon», $k^{w} a$-ítì «fille», ठi-ținga «garçon adolescent $», k^{w} a-t s i^{n} g a$ « fille adolescent », etc.) ;

- les noms de profession (ex. : ði-tyuki « docteur », ði- ${ }^{n} d \xi i f e ~ «$ policier », etc.) ;

- des types particuliers de personnes (ex. : ði-ffakù « citadin », esp. catrín);

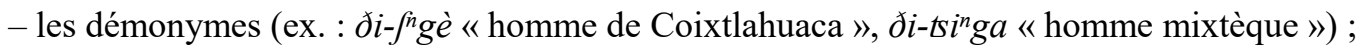

- les noms propres (ex. : ði-tfánú « Feliciano », ði-tiópa « Teófilo », kwa-liúbá « Leobarda »).

Ils sont aussi utilisés dans une fonction d'individualisation, par exemple avec les nombres cardinaux : $\partial i$ júhufi « le deuxième (homme) ».

Les termes de parenté utilisent les classificateurs humains mais de façon plus restrictive. Les deux classificateurs ne sont utilisés que dans la terminologie de parenté de la génération d'ego et les générations descendantes ; ils ne sont pas utilisés pour les générations ascendantes. Alors que le classificateur de « personne de sexe féminin » peut apparaître avec n’importe quel terme de parenté de ces générations, celui de «personne de sexe masculin » n'apparait généralement qu'avec les termes de parenté qui sont des mots empruntés à l'espagnol. Ainsi, pour beaucoup des termes de parenté il existe une opposition privative entre ceux marqués par le classificateur de «personne de sexe féminin » et ceux qui ne le sont pas ${ }^{15}$. Exemples :

- terminologie de parenté de la génération d'ego: kiffühu «frère, enfant de même parent ", $k^{w} a$ kitfühu « soeur »;

- terminologie de parenté de la génération d'ego empruntée à l'espagnol : Ji-primú «cousin» (esp. primo), $k^{w} a$-prímá « cousine» (esp. prima), di-kunáðú «beau-frère» (esp. cuñado), $k^{w} a-$ kunáðá « belle-soeur » (esp. cuñada) ;

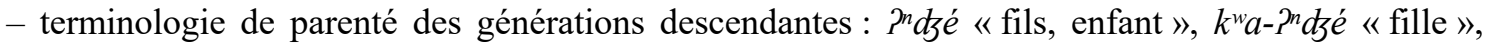
$\int k a ́ P^{n} d a$ « beau-fils », $k^{w} a-\int k a^{2 n} d a$ « belle-fille », etc.

Le classificateur nominal «animal » participe systématiquement à la formation des mots qui font référence à la faune. Souvent ce processus implique la reclassification d'un autre mot ; ainsi le nom tshẽ «miel » en combinaison avec le classificateur animal, $u$-tshề, signifie «abeille». Comme les termes de classe, ces noms classifiés se présentent sous la forme d'un complexe associant un marqueur de classification nominale et un second composant qui peut être un nom, un adjectif ou un lexème conjoint. Un exemple de ce dernier cas: le lexème jè est le terme générique pour «serpent» mais il n'a pas d'autonomie syntaxique; il apparaît uniquement avec le classificateur ( $u$-jè) ou en composés (hna-jè "Cerro Culebrón »). Comme pour les termes de classe des formes de vie inanimées, les noms construits avec le classificateur « animal » peuvent former un composé avec un nom ou un adjectif afin de distinguer les taxa spécifiques des taxa génériques : $u$-je- ${ }^{n} d a ̀$ (litt. « serpent d'eau ») désigne une classe spécifique de serpent, la «culebra corredora » (probablement Salvadora intermedia) ${ }^{16}$. Le champ d'emploi de ce classificateur

\footnotetext{
${ }^{15}$ Ceci pourrait être une innovation récente inspirée de l'espagnol : dans deux vocabulaires ixcatèques réalisés en 1887 et 1893, les termes de parenté ne montrent aucun usage des classificateurs. Pour beaucoup de systèmes de parenté mésoaméricains, les distinctions de genre sont seulement lexicalisées pour les plus âgés qu'ego, voir p. ex. Lockhart pour le nahuatl (1992 : 72-85); ceci a dû être aussi le cas pour l'ixcatèque.

${ }^{16}$ Sandra Smith Aguilar observe que Salvadora intermedia est serpent non aquatique; cependant le Thamnophis cyrtopsis est aussi signalé dans la région; c'est un serpent d'eau qui a également comme motif une bande longitudinale. Il est donc possible que les deux aient été fusionnés dans la nomenclature ixcatèque.
} 
inclut un petit nombre de référents auxquels beaucoup de langues mésoaméricaines attribuent des propriétés proches de celles des animaux. Ainsi, le terme générique pour " champignon », u-ftihi, porte-t-il le classificateur animal, un phénomène que l'on retrouve dans quelques autres langues otomangues comme le chocho ${ }^{17}$. Cette classification semble faire référence aux qualités du champignon qui se rapprochent de celles de la viande. Ainsi, en tzeltal, le thème verbal -yi' fait référence au fait de manger de la viande ou des champignons (Hunn 1977: 135) et en nahuatl le mot "champignon " (nanacatl) est dérivé du mot «viande" $(\text { nacatl })^{18}$. Le classificateur animal ixcatèque apparait aussi dans quelques noms faisant référence aux phénomènes astronomiques : u-tsé (« étoile »), u-rafté («Pléiades »). Là aussi le phénomène se retrouve dans d'autres langues otomangues comme le chocho, le popoloca, le mixtèque et l'amuzgo ${ }^{19}$. La présence du classificateur animal dans ces noms est peut-être due au fait que dans le passé les phénomènes de ce genre étaient d'une certaine façon considérés comme des animés $20{ }^{20}$. Le mot $u$-t $u k$ ?e " grain de beauté » porte aussi le classificateur animal.

Une des caractéristiques des classificateurs nominaux ixcatèques qui les différencient des termes de classe est la possibilité de tracer les référents dans le discours par la reprise anaphorique du classificateur de la personne ou de l'animal sous la forme d'un pronom suffixé au verbe, à certaines expansions nominales du verbe ou encore aux adjectifs attributs dans certaines constructions. C'est ce phénomène que VeermanLeichsenring appelle « coréférence » $(2001,2004)$.

Les pronoms anaphoriques liés à l'emploi de ces classificateurs sont les suivants :

\begin{tabular}{ll}
\hline Classificateur & Pronom \\
\hline$ð i$ «omme $~$ & $=d \grave{a}$ \\
$k^{w} a-$ « femme $~$ & $=k^{w} \grave{a}$ \\
$u \ll$ animal $»$ & $=b a$ \\
\hline
\end{tabular}

Comme Veerman-Leichsenring l'a indiqué, les pronoms sont issus de la fusion historique des classificateurs et d'un *- $a$ déictique ou déterminant. Nous traitons ces marques de coréférence comme des pronoms suffixes de 3 e personne, suivant une tradition bien établie dans les études de langues otomangues. Il y a quelques bons arguments en faveur d'une telle qualification, notamment le fait que l'on trouve en situation des énoncés du type bane $=b a$ « l'animal mange » où l'animal n'est pas nommé, ce qui atteste de l'autonomisation syntaxique de $=b a$ et de sa capacité à actualiser le verbe. L'étude détaillée de ce système de reprise coréférentielle reste à faire toutefois. Il faut analyser en détail les nombreux exemples à notre disposition, selon les structures d'actance de la langue et la structure de l'information, ce qui dépasserait le cadre du présent article. On se bornera donc à donner quelques exemples simples pour illustrer le phénomène avec un verbe locatif ou intransitif (ex. 1, 2 et 3 ) et avec un verbe transitif (ex. 4, 5 et 6) :

(1.) ði-huá kipi-ða nda-nume

CL.H-Juan être-CO.H San Pedro Nodón

'Juan est à Nodón.'(PSG élicitation 2011)

\footnotetext{
${ }^{17}$ Le mot chocho pour « champignon/fungi » est $u$-suí. Un phénomène lié est le mot en popoloca d'Otlaltepec pour « charbon de maïs » (esp. huitlacoche), ku?ndui?, qui porte également le classificateur animal popoloca $k u$.

${ }^{18}$ Ce type de dérivation nominale par le redoublement est attesté par un petit ensemble de mots pairs qui inclut aussi « enfant » $($ conètl) : « poupée » (coconētl), voir Canger 1981.

${ }^{19}$ Dans le mixtèque colonial de Teposcolula, le classificateur animal (ti-/te-) apparaît dans les mots désignant « étoile » (ti-ñuu), «arc-en-ciel » (ti-cooyándi) et «tourbillon» (ti-yachi) cf. Alvarado, 1593: 25r, 181v. En amuzgo, le classificateur animal (ki5-) apparaît dans les mots désignant « étoile » (ki5-xju35) et «arc-en-ciel» (ki5- tsu5jn'a31), cf. Tapia García, 1999: 86, 84. En popoloca d'Otlaltepec, les mots pour «arc-en-ciel » (ku"dje?t $f a$ ?) et «tourbillon» ( $\left.k u-\int i h^{n} g i\right)$ prennent aussi le classificateur animal ( $k u$ v. supra). En chocho de Nativitas, les mots désignant «étoile » $u$-nutsè) et «tourbillon » (zí-zenki) portent un des deux classificateurs animal $(u-, z i-)$, mais aussi le mot «lune » $\left(u-z i i_{\delta} u\right)$.

${ }^{20}$ En principe, en nahuatl classique seuls les noms animés peuvent être fonctionnels au pluriel mais les étoiles ont aussi une forme plurielle (Launey, $1986: 505$ ).
} 
(2.) sa u-ninà kỉi-ba aku ndziiła DEF CL.A-chien est-CO.A dans maison 'Le chien est dans la maison.'(PSG élicitation 2011)
$s a^{I}=\boldsymbol{k} \boldsymbol{w a} \boldsymbol{a}^{2}-\imath \tilde{i}^{1} \quad s i^{1} s i^{2} k a^{2}-\boldsymbol{k} \boldsymbol{w} \boldsymbol{a}^{2}$
DEF-CL.F-petit se_mettre_debout-CO.3SG.F
$k i^{1}=t \tilde{a}^{2} h \tilde{u}^{I} \quad m e^{l} s a^{2}$
PROG.3S-sur table

« La fille est debout sur une table. »

(Adamou 2014 : ex. 9b, gloses par l'auteure)

(4.) „i-hiti behe-ða hngu karga jate

CL.H-enfant offre-CO.H un chargement bois

'L'enfant va faire cadeau d'un chargement de bois.'

$$
\begin{array}{lll}
\text { u-nina } & k u \text {-tseftahun-ba } & \text { ¿i-Kalin } \\
\text { CL.A-chien } & \text { PERF-faire_peur-CO.H } & \text { CL.H-Galdino } \\
\text { 'Le chien a effrayé Galdino.' } &
\end{array}
$$

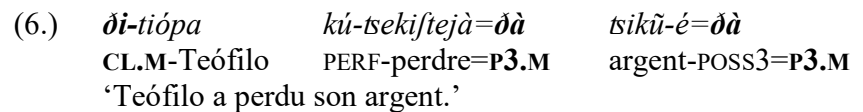

\subsection{Différences entre les classificateurs nominaux humain et animal}

Malgré leurs similitudes, le classificateur ixcatèque «animal » et les classificateurs humains diffèrent par quelques aspects importants. À la différence de $\partial i$ ou $k^{w} a$-, le monème $u$ peut encore fonctionner en tant que nom, comme le montre l'exemple suivant :

$$
\begin{aligned}
& k^{w} \text {-áhí } h^{n} g u \quad \boldsymbol{u} \\
& \text { PERF-passer un animal } \\
& \text { 'Un animal est passé.' }
\end{aligned}
$$

Toutefois de tels usages sont rarissimes et limités aux contextes où le nom n'est pas déterminé par un défini ; dans le cas général l'espèce est spécifiée avec précision et les locuteurs de l'ixcatèque ont, ou avaient, à leur disposition un lexique considérable dans ce domaine. Les lexèmes qui se combinent avec ce classificateur sont donc des noms composés dans lesquels le classificateur de nom générique est le constituant nucléaire. La relation sémantique entre ce constituant et le second composant est du type hyperonyme / hyponyme.

D'autre part, les principaux classificateurs humains ne sont pas des noms dans la mesure où ils n'ont pas d'indépendance syntaxique. De plus, la relation ontologique entre ces classificateurs et le lexème qu'ils marquent n'est pas générique-spécifique mais plutôt de genre-instance. Par exemple, đi-tfánú n'indique pas qu'il y a une "personne de sexe masculin» du type spécifique appelé "Feliciano", mais plutôt que l'instance de «Feliciano » est du « sexe masculin ». Pour cette raison, les noms propres peuvent apparaître sans classificateur nominal, par exemple dans les constructions vocatives. C'est cette même caractéristique individualisante qui permet au classificateur de «personne de sexe féminin »-mais en principe pas celui de «personne de sexe masculin »-d'apparaître dans certains termes de parenté. Les classificateurs nominaux humains ne sont pas donc des noyaux super-ordonnés des mots dans lesquels ils apparaissent.

Le classificateur de nom «animal» occupe donc une position intermédiaire par rapport aux termes de classe et aux classificateurs nominaux humains. Ses emplois comme classificateur résultent de la nécessité de pouvoir tracer le référent animé (animal) dans le discours comme possesseur, agent, bénéficiaire, etc., et le distinguer ainsi des animés humains. D'un autre côté, s'agissant d'un référent non humain, il n'y a pas de besoin lexical pour l'instanciation individuelle (les animaux n'ont pas de professions, noms, ville d'origine ou des relations de parenté qui ont besoin d'être indiquées lexicalement) et par conséquent, la relation hyperonyme / hyponyme suffit. Ce procédé de création de mot rapproche donc le classificateur «animal » des termes de classe. 


\subsection{Le quasi-classificateur}

On pourrait identifier un quasi classificateur, mi- «personne, gens», qui se trouve en relation paradigmatique avec les classificateurs nominaux humains: ठi- ${ }^{n} d a w a$ et $m i{ }^{n} d a w a$, qui veulent dire " homme » et $k^{w} a-t ? a$ et $m i-t ?$ ? $a$, signifiant tous deux «femme ». Cependant, il n'apparaît que dans une poignée de mots ; outre les deux mots ci-dessus, on trouve les exemples suivants : mi-tíut Catarina Ocotlán», mi-ina "gens riche », mi-tuhna "gens de la Sierra Mazateca ». Il semble que les pronoms puissent reprendre anaphoriquement des noms classifiés avec le quasi-classificateur, mais c'est un point qui demande une enquête plus approfondie :

$$
\begin{array}{llll}
\boldsymbol{m i}-t \text { P } a & b-i f i a k u=\boldsymbol{k}^{w} \boldsymbol{a} & \text { la=inà } & k i \text { - } f a^{n} g a=\boldsymbol{k}^{w} \grave{\boldsymbol{a}} \\
\text { QC-femme } & \text { IPRF-pense-P3.F } & \text { SUB=bien } & \text { PROG.3-être.intelligent-CO.F } \\
\text { 'La femme pense qu'elle est bien intelligente.' } &
\end{array}
$$

Il y a une parenté nette entre mi- et le nom tfahmi «gens» (Veerman, 2004: 427, 433, suivant Gudschinsky, 1959: no. 198, reconstruit $* * \check{c} a-/ \check{c} u$ - $h m i$ en protopopolocan). Il se peut que le quasi-

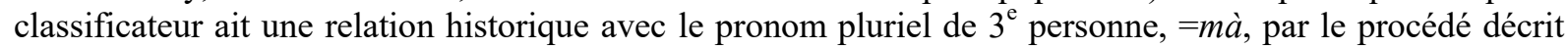
ci-dessus, mais la reprise pronominale régulière dans les énoncés serait tombée en désuétude.

\subsection{La classification des défunts}

Les personnes décédées, indépendamment de leur sexe, sont classifiées avec le nom animà, emprunt au vocabulaire de la religion catholique. Lorsqu'il est utilisé indépendamment, ce nom signifie «cœur», " âme » ou "défunt ». Comme marqueur de classification, il semble être utilisé exclusivement avec des noms propres. Ce marqueur ne se concatène pas avec les classificateurs nominaux humains; les locuteurs rejettent, par exemple, des constructions comme *aníma ði-huấ (*« feu CL.M-Juan »). Ce marqueur de classification ne permet pas non plus la reprise anaphorique ; si les locuteurs acceptent les énoncés 9 et 10 , ils rejettent sans équivoque 11 :

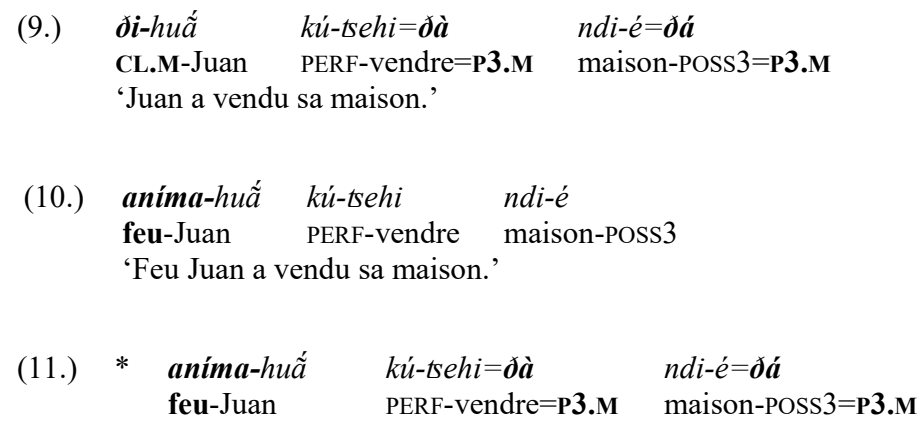

Selon les données disponibles, cette classification n'est pas présente dans les autres langues popolocanes actuelles, mais il existait un classificateur « défunt» en vieux chocho et en popoloca ${ }^{21}$.

\footnotetext{
${ }^{21}$ En vieux chocho les noms de défunts portent l'élément préposé su-ce qui correspond à chu- en vieux popoloca. L'utilisation de monèmes spéciaux pour les défunts se retrouve dans d'autres langues mésoaméricaines de l'époque coloniale ; voir par exemple la description par Fray Antonio de los Reyes du monème mixtèque colonial $\tilde{n} u$ - (Reyes, $1593: 15 \mathrm{r}$ ).
} 


\section{NOMS COMPLEXES INTÉGRÉS AU LEXIQUE VS NOMS COMPLEXES}

\section{FORMÉS EN DISCOURS}

Il convient de faire une distinction au sein des noms complexes qui nous intéressent en fonction de leur mode de formation.

a) Les noms complexes désignant des animaux et des plantes, certains objets, des liquides, des parties du corps et des toponymes sont complètement lexicalisés.

b) À l'inverse, la désignation des personnes impliquant les marqueurs de classification $\partial i$-, $k^{w} a$ - et anímà se construit en discours. Elle fait appel à deux types d'informations :

- Celle portée par le marqueur de classification : personne vivante (avec indication du sexe pour les adultes seulement) vs personne décédée (pas de mention du sexe);

- Celle portée par le second composant : anthroponyme (prénom ou nom de famille), statut social ou professionnel.

Ainsi, différentes combinaisons sont possibles pour un même anthroponyme et sont réalisées en contexte :

sikiél > ði-sikiél « monsieur Ézéquiel »

sikiél > anima sikiél « feu Ézéquiel »

wanita $>k^{w} a$-wanita « madame Juanita »

wanita > aníma wanita « feue Juanita »

Il faut noter que l'anthroponyme peut être employé seul comme vocatif dans un usage familier, les formes en $\partial i$ - ou $k^{w} a$ - servant à la citation ou comme terme d'adresse respectueux. La même chose se produit avec les noms de fonctions sociales, avec la possibilité de faire varier le sexe :

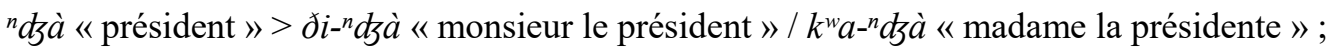

¡i-tfuki « monsieur le docteur » / $k^{w} a-t f u k i$ « madame la doctoresse ».

Toutefois, certains noms classifiés en $\partial i, k^{w} a$ - et $m i$-, construits sur le modèle marqueur de classification humain + adjectif, sont stabilisés dans le lexique :

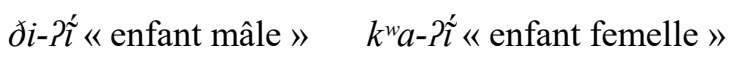

$$
\begin{aligned}
& \text { CL.M }+ \text { «petit » CL.F + « petit » } \\
& \text { ði-n dawa « homme » mi-ndawa « homme » } \\
& \text { CL.M + «mâle » quasi-classificateur + «mâle » }
\end{aligned}
$$

Dans ce contexte, ces marqueurs de classification forment un micro-paradigme qui autorise la construction de complexes variés à partir d'un même composant adjectival ${ }^{22}$.

\section{ORIGINE DES CLASSIFICATEURS NOMINAUX HUMAINS}

Il s'agit de $\Varangle i$ - «personne de sexe masculin », $k^{w} a-$ «personne de sexe féminin », auxquels on peut ajouter le quasi-classificateur $m i$ - " personne, gens ». Ces éléments qui dérivent diachroniquement de noms ont perdu l'essentiel des compatibilités syntaxiques spécifiques de cette classe sans en acquérir de nouvelles : on peut maintenant les considérer comme de simples composants synthématiques. L'unité $\partial i$ - a un sens, mais les locuteurs consultés rejettent les syntagmes du type *sa=ði (cible «l'homme, le monsieur »), ou

\footnotetext{
${ }^{22}$ On peut signaler au passage le nom $l i$-Pí « enfant », peut-être construit sur la base d'un élément * $l i$ - « enfant » et de l'adjectif « petit ». L'élément $l i$-, très peu productif, a peut-être été un marqueur de classification au même titre que $m i-$, par exemple, et serait tombé en désuétude.
} 
*útfá ði (cible «beaucoup d'hommes ») et c'est le complexe mi- ${ }^{n} d a w a$ « humain mâle » qui est utilisé en relation avec l'indéfini, le défini, les quantifieurs, les adjectifs, etc. (sa=mi-ndawa, útfá mi- $\left.{ }^{n} d a w a\right)$. De même, si $k^{w} a$ - a le sens «femme, madame », il n'apparaît pas en dehors des noms composés (? juhu $k^{w} a$, cible «deux femmes», est douteux pour les locuteurs) et c'est mi-t??a «humain femme» ou $k^{w}$ ? $a$ « épouse 》 (forme évidemment apparentée) qui sont employés comme nom. L'unité mi- «personne, humain » n'est pas non plus attestée seule. Il faut signaler cependant le cas des constructions avec le démonstratif distal -ra, du type ði-ra «celui-là, ceux-là », mi-ra «ceux-là » (mais * đi-ri, *mi-ri avec le proximal ne sont pas attestés), ces complexes, rares, étant eux-mêmes déterminables par le défini $s a$ et un quantificateur comme $k a$ « tous » (voir exemple ci-dessous), alors que *ka $\Varangle i$ ne semble pas possible. On a sans doute intérêt à analyser ces constructions avec - $r a$ comme des figements à statut de pronom démonstratif.

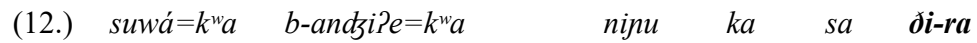 3SG-CO.F PERF-demander=CO.F tortilla tous DEF CL.H-DEM.DIST} 'Elle demande toujours des tortillas à tous ceux-là.'

L'origine des classificateurs nominaux humains n'est pas tout à fait claire. Le classificateur $k^{w} a-$ " femme (familier), madame» pourrait provenir de la forme ${ }^{*} k^{w} a ? a$ reconstruite avec le sens de «tante» en protopolocan (Gudschinsky, 1959 : no. 84, Harvey, 1963, Merrifield, 1981), et le cognat $k^{w} a$ ?a "tante » existe d'ailleurs en ixcatèque contemporain (correspondant à $k^{w} h a^{l} a^{3}$ en popoloca d'Atzingo, $\beta a ? a$ en chocho, ${ }^{*} n t u^{2}{ }^{\prime} k^{w} h \tilde{a}^{2}$ en proto-mazatèque selon Kirk, $1966:$ n. 307). Mais Veerman-Leishenring (2004: 434) donne au **khua du proto-popolocan la valeur de «femme mariée », qui se serait donc maintenue uniquement en ixcatèque. Une forme apparentée $k^{w} ? a$ a la valeur de «femelle » quand elle est employée comme modificateur (utitú $k^{w}$ ?a « chatte ») et signifie " épouse » dans des syntagmes possessifs du type $k^{w}$ Pa-nanà «mon épouse ». On peut probablement proposer pour l'ixcatèque un schéma d'évolution plus proche de ce que l'on observe dans les langues voisines. On sait que le terme esp. tía « tante » est employé couramment comme vocatif dans les régions rurales du Mexique, et à Ixcatlán, pour désigner toute femme de la communauté plus âgée qu'ego (tía Rufina, tía Iguacia...) : on peut faire l'hypothèse qu'un mécanisme de même nature a joué en ixcatèque et que le terme $k^{w} a$ ? $a$ " tante » de terme d'adresse respectueux, est passé progressivement à désigner toute femme adulte et, par un processus d'attrition formelle, a donné le classificateur $k^{w} a$. Le schéma ci-dessous illustre cette évolution possible :

Figure 1 : Développement possible du classificateur $k^{w} a$ - et du pronom suffixe $-k^{w} a$ à partir du nom « tante »

$$
\begin{aligned}
& \text { Nom } \rightarrow \text { Vocatif } \rightarrow \text { Classificateur } \rightarrow \text { Pronom }
\end{aligned}
$$

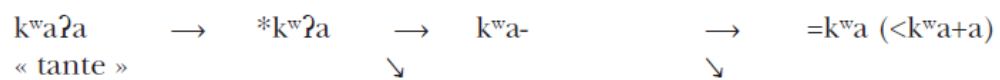

$$
\begin{aligned}
& \text { «tante » } \\
& \text { «femelle» «femme» }
\end{aligned}
$$

Nous suggérons que le même type de développement s'est produit pour le classificateur $\partial i$ - « homme » et le pronom suffixe correspondant -ða, à partir du nom tsina «oncle», qui a les cognats sil ne- en popoloca d'Atzingo et sní- en chocholtèque ; Kirk (1966: n. 34) reconstruisant ${ }^{*} \Delta i^{2}{ }^{2} n i^{24}$ en proto-mazatèque.

Figure 2 : Développement possible du classificateur $\partial i$ - et du pronom suffixe -ða à partir du nom « oncle »

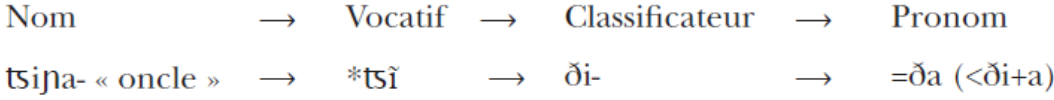

$$
\begin{aligned}
& \text { tริ̃ } \\
& \text { «mâle» «homme » }
\end{aligned}
$$


Toutefois, le changement $t s>\partial$ n'a pas encore été décrit pour l'ixcatèque ${ }^{23}$. La consonne interdentale n'apparaît que dans très peu de mots ( $\partial i^{n} g a ̀$ 'va-t'en'; $\partial i^{n} g i$ 'colline'; raðí « vieille », u-rað $i^{n} d i \grave{a}$ " renard »), presque toujours devant $i$ en syllabe non accentuée ${ }^{24}$. Compte tenu de la rareté de $\partial$, le nombre de mots apparentés est très faible. Cependant, quelques observations peuvent être faites. Une innovation partagée importante qui regroupe le chocho et le popoloca -mais exclut l'ixcatèque- est le changement *pPn $t s>t s$ (Hamp, 1958, 1960). Des développements ultérieurs du chocho ont entraîné le changement ts $>$ z dans certains contextes. Si le $\partial$ ixcatèque vient de $t$, on doit donc s'attendre à des correspondances entre ixc. $\partial$ et les consonnes rétroflexes en popoloca et chocho, ce qui se vérifie (ch. zi-zîinndià « renard »; p. -tşi « vieux, grand-parent »). Cette reconstruction -bien que provisoire en raison d'un manque de mots apparentés et d'écrits anciens- présente un cas intéressant de création de classificateurs nominaux à partir des termes de parenté, phénomène peu étudié mais peut-être pas rare. Cette hypothèse a aussi des conséquences importantes sur l'évaluation de l'ancienneté de la classification nominale humaine au sein des langues popolocanes. Veerman-Leichsenring (2004) avance que ce système remonte au protopopolocan, mais elle se fonde sur un très petit nombre de cognats : proto-popolocan *khua "(familiar) married woman»: popoloca thà-, ixcatèque $k w a-$; *ty?i «(familiar) married boy»: popoloca $t ? P^{\prime} i$-, ixcatèque $\partial i$-, protomazatèque $t i^{3}$. Sa reconstruction repose en fait sur l'idée que les mots apparentés signifiant «tante» auraient pour origine le proto-popolocan *khua «femme mariée». De plus, la reconstruction du classificateur « familiar married boy » est basée sur une correspondance de $\mathrm{pMz} * t$ : ixc. $\partial:$ p. $t$ ' qui n'est pas attestée par ailleurs. La reconstitution de ces formes est donc fortement spéculative. En fait, il n’y a aucune preuve à l'heure actuelle de l'existence de classificateurs nominaux humains en proto-popolocan. Cela devrait nous conduire à examiner de plus près les positions qui soutiennent que, dès les premiers stades, les langues otomangues possédaient de tels classificateurs (cf. Hopkins, 2012).

\section{CONCLUSION}

Dans cet article on a tenté de montrer qu'il est possible d'analyser le système de classification nominale de l'ixcatèque en fonction de différents critères. Nos observations sont les suivantes :

1) Seules certaines parties du lexique sont concernées par la classification nominale, mais le nombre des noms classifiés est important dans la langue, notamment dans le champ des dénominations de la faune et de la flore.

2) On a établi une distinction principale entre une classe «semi-fermée » des marqueurs de classification qui participent à la lexicogenèse (termes de classe), sans autre effet syntaxique ou morphologique dans la phrase, et une classe fermée de marqueurs qui ont des répercussions au-delà du nom lui-même (classificateurs nominaux).

3) On a établi une distinction principale entre les noms classifiés dont le premier composant est un marqueur spécialisé (classificateurs nominaux humains et le quasi-classificateur) et ceux dont le premier composant est un nom (terme de classe, le classificateur nominal d'animaux et le classificateur de défunts).

4) Les noms classifiés se répartissent, selon leurs compatibilités syntaxiques, entre la classe des noms et celle des noms propres.

\footnotetext{
${ }^{23}$ Le changement d'une dentale stridente sourde en interdentale fricative sonore est bien attestée dans la région et est peut-être une caractéristique de l'aire. Gudschinsky $(1959: 31)$ a posé un changement $*_{S}>\partial$ en chocho et Josserand (1983) a proposé un changement similaire dans de nombreuses variétés du mixtèque du nord de la Mixteca Alta, toutes parlées dans la région d'Ixcatlán. Le même changement est survenu dans la langue mixtèque voisine, le cuicatèque (Longacre, 1957, Rensch, 1976) et, curieusement, en zapotèque de la Sierra Juárez, le *s proto-zapotèque a changé en $\partial$ (Smith-Stark, 2007: 100).

${ }^{24}$ On ne connait que deux exemples (qui ne sont pas des emprunts) où $\partial$ apparaît devant une autre voyelle : le pronom suffixe - $a$, qui, comme Veerman-Leichsenring l'a montré est issu de la fusion du classificateur $\partial i$ - et d'un - $a$ déictique ou déterminant, et le toponyme tsaka-ndadétfi, «Barranca Ndadechi ».
} 
5) Du point de vue de la construction des noms classifiés, on a observé qu'un procédé synthématique fréquent est la combinaison d'un marqueur de classification et d'un « lexème conjoint », c'est-à-dire une unité significative sans autonomie syntaxique.

6) Certains noms classifiés, notamment ceux qui désignent la flore, peuvent entrer dans des composés à plus de deux termes de classe (concaténation), ce qui est impossible pour les classificateurs nominaux.

7) Du point de vue sémantique, on observe que la désignation des animaux et des plantes, mais également de certains objets et d'une part importante des toponymes, est fondée essentiellement sur le modèle Hypéronyme / Hyponyme ; la désignation des humains fonctionne plutôt par la qualification d'une instance selon le sexe. Certains objets peuvent être classifiés en référence à la matière (bois par exemple) ou la forme.

On résumera ces observations dans le tableau suivant :

Tableau 2 : Récapitulatif des caractéristiques des noms classifiés de l'ixcatèque

\begin{tabular}{|c|c|c|}
\hline \multicolumn{2}{|c|}{ Classificateurs nominaux } & Termes de classe \\
\hline \multicolumn{2}{|c|}{ Classe fermée } & Classe « semi-fermée » \\
\hline $\begin{array}{l}\text { Classificateurs } \partial i-, k^{w} a- \\
\text { (marqueurs spécialisés) }\end{array}$ & $\begin{array}{l}\text { Classificateur } u \text { - } \\
\text { (nom) }\end{array}$ & $\begin{array}{l}\text { Termes de classe } \\
\text { (noms) }\end{array}$ \\
\hline \multicolumn{2}{|c|}{ Coréférence $\left(-ð a,-k^{w} a,-b a\right)$} & Pas de coréférence \\
\hline $\begin{array}{l}\text { Type sémantique : } \\
\text { Sexe / Anthroponyme ou nom } \\
\text { de fonction }\end{array}$ & $\begin{array}{l}\text { Type sémantique : } \\
\text { Hyperonyme/Hyponyme }\end{array}$ & $\begin{array}{l}\text { Types sémantiques : } \\
\text { Hyperonyme/Hyponyme, } \\
\text { Méronyme/Holonyme, } \\
\text { Forme/Objet, etc. }\end{array}$ \\
\hline $\begin{array}{l}\text { Non concaténation } \\
\text { des marqueurs de classification }\end{array}$ & & $\begin{array}{l}\text { Concaténation possible et } \\
\text { courante des marqueurs } \\
\text { de classification }\end{array}$ \\
\hline
\end{tabular}

Il nous semble que ce genre d'approche présente l'avantage de ne pas couper la réflexion sur la classification nominale du fonctionnement général de la langue: il s'agit de définir quelles sont les ressources linguistiques mobilisées pour créer les noms classifiés et pour les faire fonctionner en énoncé. Le mode d'analyse utilisé nous paraît à même de rendre compte du fonctionnement réel de la classification nominale dans les langues otomangues, qui mérite d'être réexaminé selon les critères que nous proposons. La complexité des processus linguistiques impliqués suggère que le système de classification de l'ixcatèque s'est construit progressivement, en utilisant différentes ressources linguistiques. Les données comparatives et étymologiques suggèrent que les classificateurs nominaux humains en ixcatèque sont une innovation et ne peuvent être reconstruits au niveau protopopolocan. 


\section{Références bibliographiques}

Adamou Evangelia, 2014, L'antipassif en ixcatèque, Bulletin de la Société de Linguistique de Paris, 109 (1), p. 373-396.

Alvarado Francisco de, 1593, Vocabulario en lengua mixteca, Mexico, Pedro Balli.

Aikhenvald Alexandra Y., 2000, Classifiers: A typology of noun categorization devices, Oxford University Press.

Allan Keith, 1977, Classifiers, Language, 53, p. 285-311.

Antonio Ramos Pafnuncio, 2013, Los aparentes clasificadores nominales en el zapoteco de San Pedro Mixtepec (ZSPM), La Linguistique, 49-2, p. 33-50.

Aronoff Mark, 1976, Word Formation in Generative Grammar, Cambridge, MIT Press.

Austin Krumholz Jeanne, Marjorie Kalstrom Dolson \& Miguel Hernández Ayuso, 1995, Diccionario popoloca de San Juan Atzingo, Puebla, Tucson, SIL.

Beam de Azcona Rosemary, (en préparation), Grammar of Coatec Zapotec, Berlin, Mouton de Gruyter.

Belmar Francisco, 1899, Idiomas indígenas del estado de Oaxaca. El Chocho, Oaxaca.

Belmar Francisco, 1921, Glotología indígena mexicana. Estudio comparativo y clasificación de las lenguas indígenas de México, México.

Berlin Brent, 1992, Ethnobiological Classification. Principles of Categorization of Plants and Animals in Traditional Societies, Princeton, Princeton University Press.

Canger Una, 1981, Reduplication in Nahuatl, in dialectal and historical perspective, in Frances Karttunen (ed), Nahuatl Studies in Memory of Fernando Horcacitas, Texas Linguistic Forum 18, p. 29-52.

Contini-Morava Ellen \& Kilarski Marcin, 2013, Functions of nominal classification, Language Sciences, 40, p. 263-299.

Corbett Greville G. 1991, Gender, Cambridge, Cambridge University Press.

Corbett Greville G. \& Alfred D. Mtenje, 1987, Gender Agreement In Chichewa, Studies in African Linguistics, Volume 18, Number 1, p. 1-38.

Craig (Grinevald) Colette (ed.), 1986a, Noun Classes and Categorization, Amsterdam, John Benjamins, Typological Studies in Language 7.

Craig (Grinevald) Colette, 1986b, Jacaltec noun classifiers: A study in grammaticalization, Lingua, 70, p. 241-84.

Creissels Denis, 1999, 'Genres' indo-européens et 'classes nominales' Niger-Congo, Faits de langues, 14, p. 177-184.

De León Pasquel María de Lourdes, 1987, A dialectal view of Mixtec noun classifiers: productivity and fossilization, in Scott DeLancey \& Russell S. Tomlin (eds), Proceedings of the Second Annual Meeting of the Pacific Linguistics Conference, University of Oregon, p. 337-362.

De León Pasquel María de Lourdes, 1988, Noun and Numeral Classifiers in Mixtec and Tzotzil: A Referential View, PhD dissertation, University of Sussex.

DiCanio Christian (soumis), The phonetics of word-prosodic structure in Ixcatec.

Dixon Robert M. W., 1965, What is language? A new approach to linguistic description, London, Longmans.

Dixon Robert M.W., 1982, Noun classifiers and noun classes, in Dixon R.M.W., Where have all the adjectives gone?, La Hague, Mouton, p. 211-233.

Dixon Robert M.W., 1986, Noun classes, Lingua, 21, p. 104-125.

Fernández de Miranda María Teresa, 1951, Reconstrucción del protopopoloca, Revista mexicana de estudios antropológicos, 12, p. 61-93. 
Fernández de Miranda María Teresa, 1961, Diccionario ixcateco, México, INAH.

González Casanova Pablo, 1925, Los idiomas popolocas y su clasificación, Anales del Museo Nacional de Arqueología, Historia y Etnografia, 4a época, 3, p. 487-536.

Greenberg Joseph H, 1978, How does a language acquire gender-markers?, in Joseph H. Greenberg (ed.), Universals of human language, vol. 3, Word structure, Stanford, Stanford University Press, p. 47-82.

Grinevald Colette, 1999, Typologie des systèmes de classification nominale, Faits de langues, $\mathrm{n}^{\circ} 14$, p. 101-122.

Grinevald Colette, 2000, A morphosyntactic typology of classifiers, in Senft Günter (ed.), Systems of Nominal Classification, Cambridge, Cambridge University Press, p. 50-92.

Grinevald Colette, 2002, Making sense of nominal classification systems. Noun classifiers and the grammaticalization variable, in Wischer Ilse \& Gabriele Diewald (eds), New Reflections on Grammaticalization, Amsterdam/Philadelphia, John Benjamins Publishing Company, p. 259-275.

Gudschinsky Sarah C., 1956, Proto-Mazatec Structure, MA thesis, University of Pennsylvania.

Gudschinsky Sarah C., 1959, Proto-Popotecan. A Comparative Study of Popolocan and Mixtecan, Bloomington, Indiana University Publications in Anthropology, Folklore and Linguistics, 15.

Hamp, Eric, 1958, Protopopoloca internal relationships, International Journal of American Linguistics, 24 , p. $150-53$.

Hamp, Eric, 1960, Chocho-Popoloca innovations, International Journal of American Linguistics, 26 , p. 62.

Harvey, Herbert R., 1963, Términos de parentesco en el otomangue, México, INAH.

Hopkins, Nicholas. 2012. The noun classifiers of Cuchumatán Mayan languages: a case for diffusion from Otomanguean, International Journal of American Linguistics, 78, p. 411-27.

Hunn Eugene S., 1977, Tzeltal Folk Zoology. The Classification of discontinuities in Nature, New York, Academic Press.

Josserand Kathryn, 1983, Mixtec Dialect History: Proto-Mixtec and Modern Mixtec Text, PhD dissertation, Department of Anthropology, Tulane University.

Kilarski, Marcin, 2013, Nominal Classification. A history of its study from the classical period to the present, Amsterdam/Philadelphia, John Benajamins.

Kirk Paul L., 1966, Proto-Mazatec Phonology, PhD dissertation, University of Washington.

Launey Michel, 1986, Catégories et opérations dans la grammaire nahuatl, thèse d'Etat, Université de Paris IV.

Lázaro Carreter Fernando, 1968 [1953], Diccionario de términos filológicos, Madrid, Gredos

Lockhart James, 1992, The Nahuas After the Conquest: A Social and Cultural History of the Indians of Central Mexico, Sixteenth Through Eighteenth Centuries, Stanford, Stanford University Press.

Longacre Robert, 1957, Proto-Mixtecan, Bloomington, Indiana University, Indiana University Publications in Anthropology, Folklore and Linguistics, 5.

Macaulay Monica, 1996, A Grammar of Chalcatongo Mixtec, University of California Press.

Martinet André (dir.), 1979, Grammaire fonctionnelle du français, Paris, Didier-CRÉDIF.

Martinet André, 1985, Syntaxe générale, Paris, A. Colin.

Merrifield William R., 1981, Proto Otomanguean Kinship, Dallas, SIL.

Mock Carol, 1977, Chocho de Santa Catarina Ocotlán, Archivo de lenguas indígenas de México 4, México, Centro de Investigación para la Integración Social/Colegio de México/SIL.

Rebuschi Georges, 1999, Classes nominales et genre dans les langues Bantoues, Faits de langues, 14, p. 185-196.

Rensch Calvin R., 1976, Comparative Otomanguean Phonology, Language Science Monographs volume 14, Bloomington, Indiana University Publications. 
Reyes Antonio de los, 1593, Arte en lengua mixteca, Mexico, casa de Pedro Balli.

Senft Günter (ed.), 2000, Systems of Nominal Classification, Cambridge, Cambridge University Press.

Smith-Stark Thomas, 2007, Algunas isoglosas zapotecas, in C. Buenrostro et al. (eds), Clasificación de las lenguas indígenas de México. Memorias del III Coloquio Internacional de Lingüistica Mauricio Swadesh, Mexico, Instituto de Investigaciones Antropológicas-Universidad Nacional Autónoma de México / Instituto Nacional de Lenguas Indígenas, p. 69-133.

Stark, Sharon, 1978, Gramática del popoloca. Popoloca de San Marcos Tlacoyalco, México, SIL.

Swadesh Morris, 1960, The Oto-Manguean hypothesis and Macro-Mixtecan, International Journal of American Linguistics, 26, p. 79-111.

Swanton Michael, 2008, La escritura indígena como "material lingüístico": una carta en lengua ixcateca al presidente Lázaro Cárdenas, in Sebastián van Doesburg (ed), Pictografía y escritura alfabética en Oaxaca, Oaxaca, Fondo Editorial del IEEPO, p. 353-387.

Talmy Leonard, 1992, Noun, in William Bright (ed.), International encyclopedia of linguistics, vol. 3. Oxford, Oxford University Press, p. 130-31

Tapia García Fermín, 1999, Diccionario amuzgo-español : el amuzgo de San Pedro Amuzgos, Oaxaca, México, CIESAS / Plaza y Valdés.

Veerman-Leichsenring Annette, 1984, El popoloca de Los Reyes Metzontla, numéro spécial 4 de la revue Amerindia, Paris, Association d'ethnolinguistique amérindienne.

Veerman-Leichsenring Annette, 1991, Gramática del popoloca de Metzontla (con vocabulario y textos), Amsterdam, Rodopi.

Veerman-Leichsenring Annette, 1992, Semantische classificatie in het Popoloca - een historische benadering, Yumtzilob, 4(1), p. 4-15.

Veerman-Leichsenring Annette, 1994, Otomanguean languages, in E. Wyllys Andrews V \& E. Oster Mozzillo (eds.), Five Hundred Years After Columbus : Proceedings of the 47th International Congress of Americanists, New Orleans, Middle American Research Institute publication 63, p. 211-214.

Veerman-Leichsenring Annette, 2000, Popolocan independent personal pronouns: comparison and reconstruction, International Journal of American Linguistics, 66, p. 318-59.

Veerman-Leichsenring Annette, 2001a, Changes in Popolocan word order and clause structure, in J.T. Faarlund (ed.), Grammatical Relations in Change, Amsterdam, John Benjamins, p. 303-322.

Veerman-Leichsenring Annette, 2001b, Coreference in the Popolocan languages, in L.J. Brinton (ed.), Historical Linguistics 1999. Selected Papers from the 14th International Conference on Historical Linguistics, Vancouver, 9-13 August 1999, Amsterdam, John Benjamins, p. 337-350.

Veerman-Leichsenring Annette, 2001 c, Ixcateco: la frase nominal, Anales de antropología, 35, p. 323-58.

Veerman-Leichsenring Annette, 2004, Popolocan noun classifiers: a reconstruction, International Journal of American Linguistics, 70, p. 416-451.

Zavala Maldonado Roberto, 2000, Multiple Classifiers Systems in Akatek (Mayan), in Senft Günter (ed.), Systems of Nominal Classification, Cambridge, Cambridge University Press, p. 114-146.

\footnotetext{
Abréviations

1 Première personne, 3 Troisième personne, CL.A Classificateur animal, CL.F Classificateur «personne de sexe féminin », CL.M Classificateur «personne de sexe masculin », IPERF Imperfectif, P3.A pronom $3^{\mathrm{e}}$ personne « animal », P3.F pronom $3^{\mathrm{e}}$ personne « de sexe féminin », P3.M pronom $3^{\mathrm{e}}$ personne « de sexe masculin», DEF Défini, PERF Perfectif, POSS Possessif, PROG Progressif, SUB Subordonnant.
}

\section{Remerciements}

Ce travail a bénéficié de l'enquête de longue durée réalisée dans le cadre du Major Documentation Project 0214 "Textual and Lexical Documentation of Ixcatec, a Highly Endangered Otomanguean Language of Oaxaca, Mexico" (2010- 2013), financé par l'Endangered Languages Documentation Programme. Tous nos remerciements aux participants de ce projet et à nos maîtres de langue ixcatèque, Pedro, Rufina, Juliana et Cipriano. 